\title{
The search for magnetic fields in mercury-manganese stars $\star, \star \star$
}

\author{
V. Makaganiuk ${ }^{1}$, O. Kochukhov ${ }^{1}$, N. Piskunov ${ }^{1}$, S. V. Jeffers ${ }^{2}$, C. M. Johns-Krull ${ }^{4}$, C. U. Keller ${ }^{2}$, M. Rodenhuis ${ }^{2}$, \\ F. Snik ${ }^{2}$, H. C. Stempels ${ }^{1}$, and J. A. Valenti ${ }^{3}$ \\ 1 Department Physics and Astronomy, Uppsala University, Box 516, 75120 Uppsala, Sweden \\ e-mail: vitaly.makaganiuk@fysast.uu.se \\ 2 Sterrekundig Instituut, Universiteit Utrecht, PO Box 80000, 3508 TA Utrecht, The Netherlands \\ 3 Space Telescope Science Institute, 3700 San Martin Dr, Baltimore MD 21211, USA \\ ${ }^{4}$ Department of Physics and Astronomy, Rice University, 6100 Main Street, Houston, TX 77005, USA
}

Received 31 August 2010 / Accepted 12 October 2010

\begin{abstract}
Context. A subclass of the upper main-sequence chemically peculiar stars, mercury-manganese $(\mathrm{HgMn})$ stars were traditionally considered to be non-magnetic, showing no evidence of variability in their spectral line profiles. However, discoveries of chemical inhomogeneities on their surfaces imply that this assumption should be investigated. In particular, spectroscopic time-series of AR Aur, $\alpha$ And, and five other $\mathrm{HgMn}$ stars indicate the presence of chemical spots. At the same time, no signatures of global magnetic fields have been detected.

Aims. We attempt to understand the physical mechanism that causes the formation of chemical spots in HgMn stars and gain insight into the potential magnetic field properties at their surfaces; we performed a highly sensitive search for magnetic fields for a large set of $\mathrm{HgMn}$ stars.

Methods. With the aid of a new polarimeter attached to the HARPS spectrometer at the ESO $3.6 \mathrm{~m}$-telescope, we obtained highquality circular polarization spectra of 41 single and double $\mathrm{HgMn}$ stars. Using a multi-line analysis technique on each star, we co-added information from hundreds of spectral lines to ensure significantly greater sensitivity to the presence of magnetic fields, including very weak fields.

Results. For the 47 individual objects studied, including six components of SB2 systems, we do not detect any magnetic fields at greater than the $3 \sigma$ level. The lack of detection in the circular polarization profiles indicates that if strong fields are present on these stars, they must have complex surface topologies. For simple global fields, our detection limits imply upper limits to the fields present of $2-10$ Gauss in the best cases.

Conclusions. We conclude that HgMn stars lack large-scale magnetic fields, which is typical of spotted magnetic Ap stars, of sufficient strength to form and sustain the chemical spots observed on HgMn stars. Our study confirms that in addition to magnetically altered atomic diffusion, there exists another differentiation mechanism operating in the atmospheres of late-B main sequence stars that can produce compositional inhomogeneities on their surfaces.
\end{abstract}

Key words. stars: chemically peculiar - stars: magnetic field - polarization

\section{Introduction}

Mercury-manganese (HgMn) stars form a subclass of the upper main-sequence chemically peculiar $(\mathrm{CP})$ stars, that have a notable overabundance of $\mathrm{Hg}, \mathrm{Mn}, \mathrm{Y}, \mathrm{Sr}$, and other, mostly heavy, chemical elements with respect to the solar chemical composition. HgMn stars are frequently found in binaries and lie on the $\mathrm{H}-\mathrm{R}$ diagram between the early- $\mathrm{A}$ and late-B spectral types, which corresponds to $T_{\text {eff }}=9500-16000 \mathrm{~K}$ (Dworetsky 1993).

While most other, normal stars in this spectral range are rapid rotators with $v_{\mathrm{e}} \sin i=200-300 \mathrm{~km} \mathrm{~s}^{-1}$ (Abt \& Morrell 1995), $\mathrm{HgMn}$ stars are typically slow rotators, making them ideal late-B targets for detailed abundance analyses. The $\mathrm{HgMn}$ stars were for a long time considered to have chemically homogeneous atmospheres, with no obvious vertical chemical abundance stratifications or horizontal concentrations of some elements into

* Based on observations collected at the European Southern Observatory, Chile (ESO programs 083.D-1000, 084.D-0338, 085.D-0296).

$\star \star$ Figure 5 is only available in electronic form at http://www . aanda. org surface spots of high elemental abundance similar to those found in magnetic Ap stars of similar temperatures (Kochukhov 2004). This view was historically supported by the lack of a definite detection of line profile variability in any $\mathrm{HgMn}$ star. However, Adelman et al. (2002) reported variability in the spectral line of Hg II $\lambda 3984 \AA$ based on high-resolution and high signalto-noise $(S / N)$ ratio spectroscopic data obtained for the brightest HgMn star: $\alpha$ And. They attributed the variability in the Hg II line to horizontal inhomogeneities of the mercury abundance across the stellar surface and reconstructed a surface map of $\mathrm{Hg}$ with the help of Doppler imaging.

Later, Kochukhov et al. (2005) found that two other HgMn stars (HR 1185 and HR 8723) possess spotted structure in their abundance of $\mathrm{Hg}$. The fourth star, the eclipsing $\mathrm{HgMn}$ binary AR Aur studied by Hubrig et al. (2006), was reported to exhibit line profile variability for $\mathrm{Hg}$ and also such chemical elements as Y, Zr, Pt, and Sr. These results were confirmed by the independent study of Folsom et al. (2010).

An additional unexpected discovery was made by Kochukhov et al. (2007) for the $\mathrm{HgMn}$ star $\alpha$ And. From the analysis of the Hg II $3984 \AA$ line observed over a timespan 
of 7 years, they inferred surface maps that display morphological changes of spots on a timescale of only a few years. In comparison to well-studied magnetic CP stars, this behavior of the surface abundance distribution is quite surprising because all other early-type spotted stars show spot configurations that are stable over tens of years (e.g., Adelman et al. 2001).

Finally, Briquet et al. (2010) investigated time-series spectra of three more HgMn stars and concluded that all of them display variability in their line profiles. The star HD 11753 showed variability in Y, Sr and Ti; while HD 53244 and HD 221507 both show variable profiles of their $\mathrm{Mn}$ and $\mathrm{Hg}$ lines. In addition, HD 221507 displays variations in its Y lines. In summary, these studies demonstrate there are at least seven spotted HgMn stars and possibly many more. It is still not clear how typical this behavior is for this class of CP stars. Furthermore, it is not clear what physical processes are responsible for the formation of these structures on the stellar surface.

It is generally believed that a strong magnetic field is a necessary ingredient for creating inhomogeneities in the stellar atmosphere, leading, for example, to the formation of temperature or chemical spots (e.g., Jeffers \& Donati 2008; Lüftinger et al. 2010). Within this framework, a number of spectropolarimetric observations have been carried out on $\mathrm{HgMn}$ stars to confirm or disprove the presence of magnetic fields on these stars that could be responsible for the formation of chemical spots. The first systematic attempt to detect magnetic signatures in highresolution circularly polarized spectra of $\mathrm{HgMn}$ stars was made by Shorlin et al. (2002). This study included a sample of ten stars that were analyzed at a resolution of $R=35000$, yielding upper limits to the magnetic field strength of $29-100 \mathrm{G}$. To date, this is the only extensive magnetic survey of HgMn stars. Several other studies have searched for magnetic fields in individual spotted HgMn stars. For instance, Wade et al. (2006) searched for a magnetic field in $\alpha$ And. They analyzed magnetic field measurements obtained with three different polarimeters and concluded that the star has no longitudinal magnetic field stronger than about 6-19 G. Folsom et al. (2010) performed a similar analysis for AR Aur, once again finding no field stronger than 20-40 G. Finally, Auriere et al. (2010) presented an analysis of a small sample of three bright, sharp-lined $\mathrm{HgMn}$ stars, reporting no magnetic field detections at the level of 1-3 G.

Previous magnetic field studies of $\mathrm{HgMn}$ stars were affected by several fundamental limitations. First, with the exception of the four stars studied by Wade et al. (2006) and Auriere et al. (2010), previous work was not particularly precise, possibly missing weak magnetic fields which are still capable of causing chemical spot formation. Second, they included a small number of $\mathrm{HgMn}$ stars, often with a strong emphasis on the most slowly rotating ones. Thus, the class as a whole has not been well surveyed.

To overcome drawbacks associated with previous magnetic field studies on these stars, and provide new insights into the spot formation physics of late-B stars, we have carried out a spectropolarimetric survey of a large sample of $\mathrm{HgMn}$ stars. We investigate $\mathrm{HgMn}$ stars with a broad range of atmospheric parameters and rotational velocities. Most of these objects have not been studied before with the high-resolution spectropolarimetry. Taking advantage of a new polarimeter, HARPSpol, attached to the HARPS instrument at the ESO 3.6-m telescope in La Silla, Chile, we have been able to push the limits of magnetic field detection in early-type stars to remarkably low levels.

In Sect. 2.1, we describe our target selection procedure. Section 2.2 discusses our spectropolarimetric observations. Section 2.3 outlines the principles of the reduction of the spectropolarimetric data from HARPSpol. We discuss our multiline magnetic field detection technique in Sect. 3.1 and present the results of our magnetic field measurements in Sect. 3.2. Section 4 summarizes our results and discusses them in the context of studies of HgMn stars.

\section{Observations}

\subsection{Target selection}

To compile our target list, we used the catalogue of Ap, $\mathrm{HgMn}$, and Am stars published by Renson \& Manfroid (2009). To optimize our observations for the detection of weak magnetic fields and cover a statistically large sample of HgMn stars, we placed several constraints on the stellar parameters. All objects with $v_{\mathrm{e}} \sin i \gtrsim 70 \mathrm{~km} \mathrm{~s}^{-1}$ were excluded because magnetic field measurements are considerably less accurate for rapid rotators than the moderately and, in particular, slowly rotating stars. We also preferentially selected brighter stars. In attempting to reach $S / N$ of $\approx 300$, we selected only targets brighter than $m_{\mathrm{V}}=7$. A total of $45 \mathrm{HgMn}$ stars were found that satisfied these criteria. Our three observing runs covered 41 of them. Among these stars, six are spectroscopic binary systems, allowing magnetic field measurements to be performed for both components.

In Table 1, we present the list of observed stars, giving their HD and HR numbers, visual magnitude, effective temperature, and binary status. The effective temperature of all objects was estimated using the Moon \& Dworetsky (1985) calibration of the Strömgren photometric parameters as implemented in the TempLogG code (Kupka \& Bruntt 2001). The Strömgren photometry was obtained from the Simbad ${ }^{1}$ database. Information about binarity was extracted from the catalogue of Pourbaix et al. (2004) and complemented by the results of several recent studies of individual binary systems. For a few stars, we used our LSD line profiles as described below to detect binarity.

\subsection{Spectropolarimetric observations}

Our observations were obtained using the newly built polarimeter HARPSpol (Snik et al. 2008) attached to the HARPS spectrometer (Mayor et al. 2003) at the ESO 3.6-m telescope. With a resolving power of $R=115000$, this instrument is the highest resolution spectropolarimeter available to the astronomical community. The compact optical design of the polarimeter allows it to be mounted before the fiber entrance at the Cassegrain focus of the telescope. This positioning of the polarimeter minimizes the instrumental polarization that is usually caused by the oblique reflections in the light path. HARPSpol consists of two independent polarimeters for circular and linear polarization measurements. Thus, it is possible to make observations in all four Stokes parameters. Each polarimeter consists of a superachromatic retarder plate (a half-wave or a quarter-wave plate) and a beam splitter based on a Foster prism. This design makes the polarimeter fully achromatic. The two polarized light spectra are recorded simultaneously through the two HARPS entrance fibers.

Our observations were carried out in May-June of 2009, January of 2010, and April-May of 2010. The date that each of our target stars was observed is recorded in Table 2. In total, we observed HgMn targets on 26 nights, which were shared with other spectropolarimetric programs. We obtained highresolution spectra with a typical $S / N$ of $150-400$. The spectra

1 http://simbad.u-strasbg.fr/simbad/ 
Table 1. The list of HgMn stars included in our survey.

\begin{tabular}{|c|c|c|c|c|}
\hline HD number & HR number & $m_{\mathrm{V}}$ & $T_{\text {eff }}(\mathrm{K})$ & Binarity \\
\hline HD 1909 & HR 89 & 6.56 & 12406 & SB2 \\
\hline HD 11753 & HR 558 & 5.11 & 10476 & SB1 \\
\hline HD 27376 & HR 1347 & 3.55 & 12849 & SB2 \\
\hline HD 28217 & HR 1402 & 5.87 & 13906 & SB1 \\
\hline HD 29589 & HR 1484 & 5.45 & 14763 & \\
\hline HD 32964 & HR 1657 & 5.10 & 11119 & SB2 \\
\hline HD 33647 & HR 1690 & 6.67 & 12440 & SB2 \\
\hline HD 33904 & HR 1702 & 3.28 & 12759 & \\
\hline HD 34880 & HR 1759 & 6.41 & 13269 & \\
\hline HD 35548 & HR 1800 & 6.56 & 11164 & SB2 \\
\hline HD 36881 & HR 1883 & 5.63 & 10903 & SB1 \\
\hline HD 42657 & HR 2202 & 6.20 & 12842 & \\
\hline HD 53244 & HR 2657 & 4.10 & 13596 & \\
\hline HD 53929 & HR 2676 & 6.09 & 13908 & \\
\hline HD 63975 & HR 3059 & 5.13 & 13458 & \\
\hline HD 65950 & & 6.87 & 12710 & \\
\hline HD 68099 & HR 3201 & 6.08 & 12997 & \\
\hline HD 70235 & HR 3273 & 6.43 & 12329 & \\
\hline HD 71066 & HR 3302 & 5.62 & 12131 & \\
\hline HD 71833 & HR 3345 & 6.67 & 12985 & \\
\hline HD 72208 & HR 3361 & 6.83 & 11141 & SB2 \\
\hline HD 75333 & HR 3500 & 5.31 & 12248 & \\
\hline HD 78316 & HR 3623 & 5.24 & 13639 & SB2 \\
\hline HD 90264 & HR 4089 & 4.95 & 15121 & SB2 \\
\hline HD 101189 & HR 4487 & 5.14 & 11148 & \\
\hline HD 106625 & HR 4662 & 2.59 & 12002 & SB1 \\
\hline HD 110073 & HR 4817 & 4.63 & 12876 & \\
\hline HD 141556 & HR 5883 & 3.96 & 10684 & SB2 \\
\hline HD 149121 & HR 6158 & 5.62 & 11021 & SB1 \\
\hline HD 158704 & HR 6520 & 6.06 & 13378 & SB2 \\
\hline HD 165493 & HR 6759 & 6.15 & 14375 & SB2 \\
\hline HD 175640 & HR 7143 & 6.20 & 12075 & \\
\hline HD 178065 & HR 7245 & 6.56 & 12348 & SB1 \\
\hline HD 179761 & HR 7287 & 5.14 & 13010 & \\
\hline HD 186122 & HR 7493 & 6.33 & 12901 & \\
\hline HD 191110 & HR 7694 & 6.18 & 12107 & SB2 \\
\hline HD 193452 & HR 7775 & 6.10 & 10881 & SB1 \\
\hline HD 194783 & HR 7817 & 6.08 & 13803 & \\
\hline HD 202671 & HR 8137 & 5.39 & 13696 & \\
\hline HD 211838 & HR 8512 & 5.35 & 12593 & SB1 \\
\hline HD 221507 & HR 8937 & 4.37 & 12476 & \\
\hline
\end{tabular}

were recorded by a mosaic of two $2 \mathrm{~K} \times 4 \mathrm{~K} \mathrm{CCDs}$, providing 45 polarimetric echelle orders on the blue CCD and 26 on the red one. For each night, we acquired a standard set of calibration images: 20 bias exposures, 20 flat fields, and two ThAr frames. Flat-field and ThAr images were acquired with the polarimeter in the circular polarization mode.

All HgMn stars were observed in circular polarization, covering the wavelength range of 3780-6913 $\AA$ with a small gap at 5259-5337 $\AA$. Each observation of an individual star was divided into four sub-exposures corresponding to differing positions of the quarter-wave plate: $45^{\circ}, 135^{\circ}, 225^{\circ}$, and $315^{\circ}$ relative to the optical axis of the beam-splitter. The length of individual sub-exposures ranged between $160 \mathrm{~s}$ and $350 \mathrm{~s}$. In a few cases, stars were observed with only two sub-exposures. The majority of our targets were observed only once; however, ten stars, mainly spectroscopic binaries, were observed several times. For HD 11753 and HD 32964, we obtained complete coverage of the rotational and orbital periods, respectively. An analysis of these $\mathrm{HgMn}$ stars will be presented in future publications.

\subsection{Reduction of the data}

The reduction of our HARPSpol observations was performed with the REDUCE package of Piskunov \& Valenti (2002). This set of IDL routines applies a standard sequence of reduction and calibration procedures to the cross-dispersed echelle spectra. Bias images are averaged and subtracted from the average flat and science images. Spectral orders are located using the average flat field with the help of a cluster analysis method. The flat field is normalized and is used to correct the pixel-to-pixel sensitivity variations in the science images. After the removal of scattered light, the science spectra are extracted using the optimal extraction algorithm described by Piskunov \& Valenti (2002).

The intrinsic long-term stability of the HARPS spectrometer is about $1 \mathrm{~m} \mathrm{~s}^{-1}$ (Mayor et al. 2003). We do not expect the polarimeter to adversely affect this stability because it does not significantly modify the seeing-limited illumination of the HARPS fibers (Snik et al. 2008). Since we do not require an extremely high velocity accuracy for our observations, it was sufficient to use only one nightly ThAr spectrum for the wavelength calibration. Using $\approx 700-900 \mathrm{ThAr}$ lines in a $2 \mathrm{D}$ wavelength calibration routine, we obtained an internal wavelength calibration accuracy of 18-21 $\mathrm{m} \mathrm{s}^{-1}$. In the final step before the calculation of the Stokes parameter spectra, we performed continuum normalization, dividing each spectrum by a smooth, slowly varying function. This function was obtained by fitting the upper envelope of the blaze-corrected merged spectrum.

For the calculation of the circular polarization, we use the ratio method described by Bagnulo et al. (2009). The method reduces the spurious polarization effects by an appropriate combination of the two physical beams recorded for four different retarder plate positions. Along with the circular polarization, we also derive a diagnostic null spectrum. It is obtained by combining individual sub-exposures destructively, thus canceling the stellar polarization signal and showing the residual instrumental polarization remaining after application of the ratio method. Below we use the null spectrum in the same analysis steps as the Stokes $V$ spectrum, thus providing a realistic estimate of possible errors.

Using the eight spectra in the four individual sub-exposures of each star provides a convenient means of detecting and removing cosmic ray hits that otherwise seriously distort the final Stokes spectra. Affected pixels are identified by their large deviation from the median, whose value they are for. Since our exposure times were relatively short, only $1-2$ pixels required correction in each echelle order.

\section{Data analysis and results}

\subsection{LSD analysis}

A major difficulty when searching for weak (below $100 \mathrm{G}$ ) magnetic fields in stars is the weakness of the expected polarimetric signal. As one can see from Fig. 1, it is difficult to conclude anything about the presence or absence of the magnetic signal in individual spectral lines even with our highest quality Stokes $V$ spectra. To alleviate this problem, a line addition technique, called least-squares deconvolution (hereafter LSD) developed by Donati et al. (1997), is commonly used to detect weak stellar magnetic fields. This technique has proven to be a very effective tool, allowing extraction of high-precision Stokes $I$ and $V$ profiles by combining the information from all available metal lines in the spectrum. The main assumption of LSD is that all spectral lines are identical in shape and can be represented by a 
Table 2. Magnetic field analysis of $\mathrm{HgMn}$ stars.

\begin{tabular}{|c|c|c|c|c|c|c|c|}
\hline Target & Comp. & HJD- $24 \times 10^{5}$ & $S / N$ & $S / N(\mathrm{LSD})$ & $\left\langle B_{z}\right\rangle(\mathrm{V}), \mathrm{G}$ & $\left\langle B_{z}\right\rangle$ (null), G & $\mathrm{FAP} \times 10$ \\
\hline HD 1909 & $\mathrm{~A}$ & 55202.527489 & 200 & 1494 & $-4.02 \pm 7.25$ & $9.64 \pm 7.11$ & 2.1790 \\
\hline HD 1909 & B & 55202.527489 & 200 & 1494 & $137.13 \pm 58.90$ & $-61.21 \pm 57.77$ & 0.4248 \\
\hline HD 1909 & A & 55210.520641 & 178 & 1262 & $4.60 \pm 8.25$ & $-8.42 \pm 8.15$ & 1.0770 \\
\hline HD 1909 & B & 55210.520641 & 178 & 1262 & $47.95 \pm 61.14$ & $-99.79 \pm 60.43$ & 9.6940 \\
\hline HD 1909 & A & 55212.522266 & 174 & 1330 & $16.81 \pm 8.87$ & $2.77 \pm 8.76$ & 4.2570 \\
\hline HD 1909 & B & 55212.522266 & 174 & 1330 & $12.29 \pm 56.73$ & $-48.85 \pm 56.08$ & 5.6790 \\
\hline HD 27376 & $\mathrm{AB}$ & 55201.768664 & 507 & 3355 & $7.91 \pm 3.96$ & $6.82 \pm 3.94$ & 4.5860 \\
\hline HD 27376 & A & 55210.756062 & 422 & 2926 & $-0.30 \pm 5.26$ & $10.76 \pm 5.23$ & 4.0610 \\
\hline HD 27376 & B & 55210.756062 & 422 & 2926 & $-8.80 \pm 7.01$ & $-5.19 \pm 6.98$ & 2.2240 \\
\hline HD 27376 & A & 55212.741449 & 589 & 4083 & $-2.45 \pm 3.89$ & $-2.22 \pm 3.88$ & 9.3210 \\
\hline HD 27376 & B & 55212.741449 & 589 & 4083 & $4.78 \pm 4.41$ & $3.55 \pm 4.40$ & 9.9540 \\
\hline HD 27376 & A & 55213.730549 & 411 & 3071 & $5.62 \pm 4.91$ & $8.78 \pm 4.90$ & 8.4040 \\
\hline HD 27376 & B & 55213.730549 & 411 & 3071 & $1.13 \pm 6.48$ & $-12.67 \pm 6.46$ & 3.9460 \\
\hline HD 28217 & & 55213.538340 & 283 & 1472 & $-12.30 \pm 46.87$ & $46.35 \pm 46.60$ & 1.4140 \\
\hline HD 29589 & & 55201.637379 & 267 & 1302 & $-32.34 \pm 41.38$ & $-38.16 \pm 41.16$ & 0.5699 \\
\hline HD 33647 & & 55204.752546 & 177 & 1129 & $29.51 \pm 38.47$ & $-34.16 \pm 37.99$ & 3.8950 \\
\hline HD 33904 & & 55204.767578 & 600 & 4059 & $-2.55 \pm 2.59$ & $1.94 \pm 2.57$ & 4.0470 \\
\hline HD 34880 & & 55201.786748 & 154 & 933 & $55.57 \pm 49.14$ & $79.04 \pm 47.99$ & 9.2710 \\
\hline HD 35548 & & 55202.740129 & 172 & 1588 & $-0.74 \pm 2.54$ & $0.29 \pm 2.51$ & 5.0180 \\
\hline HD 36881 & & 55200.768514 & 262 & 2451 & $-7.09 \pm 5.49$ & $4.58 \pm 5.47$ & 8.1070 \\
\hline HD 42657 & & 55202.757839 & 185 & 1220 & $-30.84 \pm 79.97$ & $17.52 \pm 79.25$ & 7.4910 \\
\hline HD 53244 & & 55204.779860 & 477 & 2692 & $14.71 \pm 13.06$ & $16.05 \pm 12.98$ & 0.0655 \\
\hline HD 53929 & & 55200.792070 & 319 & 1783 & $7.14 \pm 10.77$ & $-13.22 \pm 10.59$ & 9.9250 \\
\hline HD 63975 & & 55204.793412 & 299 & 1828 & $8.28 \pm 11.55$ & $-3.83 \pm 11.34$ & 6.8330 \\
\hline HD 65950 & & 55206.754842 & 143 & 1117 & $-0.69 \pm 16.87$ & $-24.08 \pm 16.35$ & 7.2770 \\
\hline HD 65950 & & 55211.765235 & 152 & 1039 & $6.78 \pm 18.33$ & $12.50 \pm 17.77$ & 7.1000 \\
\hline HD 65950 & & 55212.797482 & 174 & 1355 & $-2.35 \pm 13.79$ & $9.05 \pm 13.51$ & 3.4760 \\
\hline HD 68099 & & 55202.773866 & 164 & 1096 & $-108.34 \pm 69.86$ & $40.66 \pm 68.10$ & 4.9430 \\
\hline HD 68099 & & 55212.821947 & 206 & 1381 & $96.70 \pm 58.42$ & $31.10 \pm 57.31$ & 6.1680 \\
\hline HD 70235 & & 55205.769179 & 178 & 1292 & $22.95 \pm 14.90$ & $2.99 \pm 14.66$ & 4.9740 \\
\hline HD 70235 & & 55212.837161 & 204 & 1581 & $30.27 \pm 11.93$ & $-14.35 \pm 11.72$ & 9.1010 \\
\hline HD 71066 & & 55205.786739 & 389 & 2730 & $-1.14 \pm 0.81$ & $0.24 \pm 0.80$ & 0.6773 \\
\hline HD 71833 & & 55205.812637 & 193 & 1253 & $27.38 \pm 26.46$ & $-14.94 \pm 25.91$ & 5.7850 \\
\hline HD 71833 & & 55212.855644 & 153 & 1171 & $-1.69 \pm 27.67$ & $20.61 \pm 27.06$ & 8.1900 \\
\hline HD 72208 & & 55202.789124 & 140 & 1289 & $61.39 \pm 38.66$ & $-48.43 \pm 38.20$ & 7.2000 \\
\hline HD 72208 & & 55209.769108 & 157 & 1445 & $-2.11 \pm 41.08$ & $-46.09 \pm 40.41$ & 0.1644 \\
\hline HD 75333 & & 55206.823570 & 192 & 2155 & $4.38 \pm 15.96$ & $-3.40 \pm 15.78$ & 3.8920 \\
\hline HD 78316 & & 55202.803837 & 230 & 1311 & $0.03 \pm 4.12$ & $0.42 \pm 4.04$ & 3.9410 \\
\hline HD 78316 & & 55211.787325 & 339 & 1926 & $-0.38 \pm 2.66$ & $-3.40 \pm 2.64$ & 5.3000 \\
\hline HD 90264 & A & 55201.850566 & 255 & 1461 & $3.43 \pm 8.39$ & $-33.70 \pm 8.23$ & 5.7250 \\
\hline HD 90264 & $\mathrm{~B}$ & 55201.850566 & 255 & 1461 & $3.34 \pm 12.42$ & $-2.50 \pm 12.17$ & 0.6511 \\
\hline HD 101189 & & 54982.974439 & 51 & 262 & $-95.22 \pm 65.63$ & $-53.75 \pm 62.58$ & 0.4069 \\
\hline HD 101189 & & 55201.862940 & 179 & 2797 & $-6.41 \pm 5.43$ & $4.52 \pm 5.37$ & 1.4030 \\
\hline HD 106625 & & 54983.067376 & 149 & 2493 & $-15.99 \pm 18.20$ & $13.06 \pm 18.41$ & 4.1200 \\
\hline HD 106625 & & 55205.872226 & 881 & 4348 & $14.71 \pm 11.11$ & $7.98 \pm 11.13$ & 10.000 \\
\hline HD 110073 & & 55205.856731 & 417 & 2813 & $2.96 \pm 4.25$ & $1.71 \pm 4.26$ & 9.9040 \\
\hline HD 141556 & A & 54982.163224 & 120 & 1613 & $-1.91 \pm 3.46$ & & 7.4680 \\
\hline HD 141556 & B & 54982.163224 & 120 & 1613 & $8.38 \pm 10.81$ & & 1.4170 \\
\hline HD 141556 & A & 55317.770736 & 143 & 1710 & $1.54 \pm 4.48$ & $-12.63 \pm 4.51$ & 2.9260 \\
\hline HD 141556 & B & 55317.770736 & 143 & 1710 & $17.90 \pm 11.08$ & $8.03 \pm 11.16$ & 1.9210 \\
\hline HD 149121 & & 55316.735917 & 217 & 1840 & $-0.02 \pm 4.45$ & $1.04 \pm 4.48$ & 6.4580 \\
\hline HD 158704 & & 55316.845983 & 172 & 967 & $-0.76 \pm 3.56$ & $-1.91 \pm 3.50$ & 2.6810 \\
\hline HD 165493 & A & 55316.925608 & 144 & 796 & $4.77 \pm 5.56$ & $-2.10 \pm 5.51$ & 8.1200 \\
\hline HD 165493 & B & 55316.925608 & 144 & 796 & $-31.05 \pm 27.67$ & $-80.86 \pm 27.42$ & 0.9936 \\
\hline HD 175640 & & 55317.865578 & 170 & 1327 & $-0.59 \pm 2.16$ & $-3.05 \pm 2.16$ & 8.4280 \\
\hline HD 178065 & & 55317.879714 & 57 & 431 & $-1.92 \pm 8.81$ & & 9.6770 \\
\hline HD 178065 & & 55319.831679 & 147 & 1323 & $-2.26 \pm 2.08$ & $-4.12 \pm 2.08$ & 0.0085 \\
\hline HD 179761 & & 55319.926735 & 123 & 1048 & $23.92 \pm 19.26$ & $-21.95 \pm 19.07$ & 5.1960 \\
\hline HD 186122 & & 55319.850529 & 203 & 1252 & $0.66 \pm 1.85$ & $0.45 \pm 1.83$ & 7.3990 \\
\hline HD 191110 & A & 55319.934190 & 143 & 1256 & $1.67 \pm 7.17$ & $4.27 \pm 7.13$ & 9.8480 \\
\hline HD 191110 & $\mathrm{~B}$ & 55319.934190 & 143 & 1256 & $6.26 \pm 9.15$ & $20.55 \pm 9.10$ & 7.2040 \\
\hline HD 193452 & & 55316.939679 & 90 & 964 & $-2.10 \pm 2.37$ & & 2.2220 \\
\hline HD 193452 & & 55319.868330 & 203 & 1868 & $-1.04 \pm 1.42$ & $-0.70 \pm 1.41$ & 2.0460 \\
\hline HD 194783 & & 54984.354843 & 160 & 1316 & $10.08 \pm 19.75$ & $-3.23 \pm 20.05$ & 8.1140 \\
\hline HD 194783 & & 55319.945379 & 136 & 1064 & $44.01 \pm 21.60$ & $24.02 \pm 21.07$ & 4.0580 \\
\hline HD 202671 & & 54984.382173 & 175 & 1220 & $25.51 \pm 16.01$ & $-25.08 \pm 16.23$ & 2.3420 \\
\hline HD 211838 & & 54984.396740 & 150 & 1432 & $-194.23 \pm 77.70$ & $-2.01 \pm 79.02$ & 7.1810 \\
\hline HD 221507 & & 55210.548529 & 349 & 2498 & $4.21 \pm 7.04$ & $-9.51 \pm 6.99$ & 9.4710 \\
\hline
\end{tabular}


V. Makaganiuk et al.: The search for magnetic fields in mercury-manganese stars

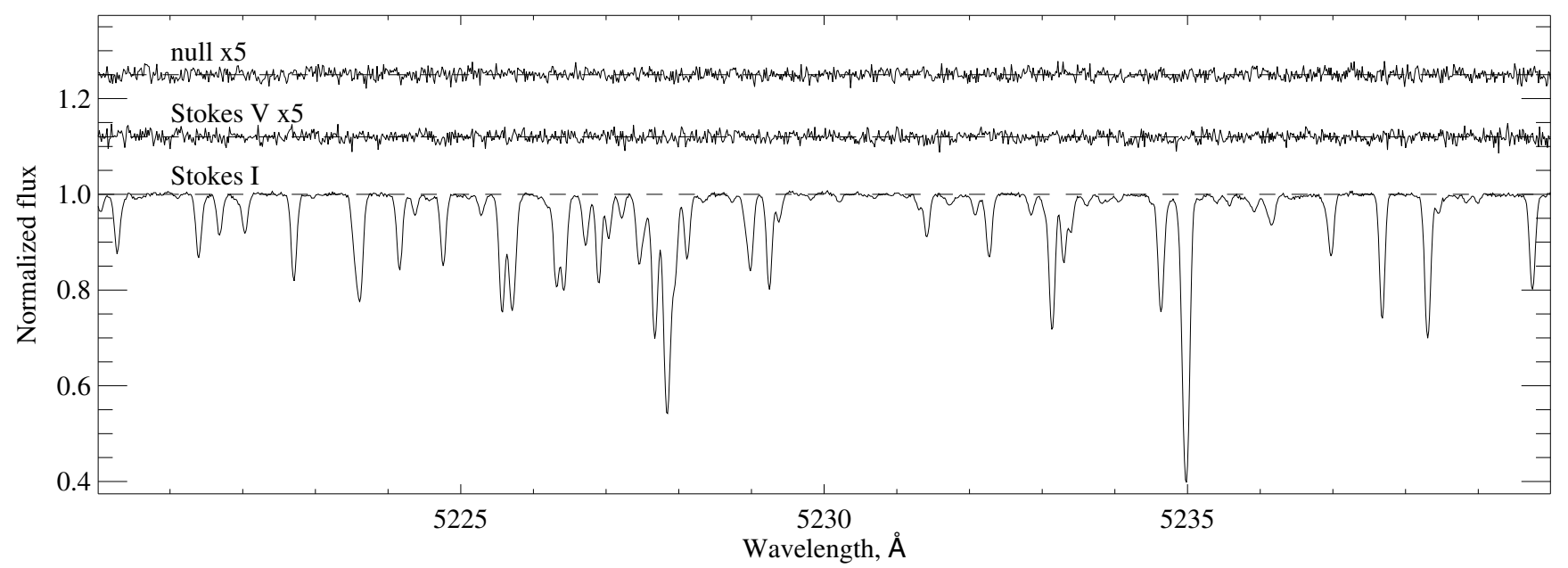

Fig. 1. The HARPSpol spectra of HD 71066 in the wavelength region of 5220-5240 A. Spectra are displayed from bottom to top as follows: Stokes $I$, Stokes $V$ and the null spectrum. Note, that for displaying purposes Stokes $V$ and the null spectrum are shifted upwards by 1.12 and 1.25 , respectively, and are multiplied by 5 .

scaled average profile. Using this model, one can reconstruct the average profile from an observed intensity or polarization spectrum, obtaining an increase in the $S / N$ ratio by a factor of up to 30-40. Previous studies have demonstrated that with the help of the LSD technique, it is possible to measure magnetic fields weaker than $1 \mathrm{G}$ (Aurière et al. 2009; Lignières et al. 2009).

The LSD analysis requires prior knowledge of the line positions and strengths. We compiled a number of line lists using VALD (Piskunov et al. 1995) together with 1D, LTE stellar atmosphere models with effective temperatures ranging from $10000 \mathrm{~K}$ to $18000 \mathrm{~K}$ in $500 \mathrm{~K}$ steps. The atmosphere models were calculated with the LLmodels code (Shulyak et al. 2004), assuming solar abundances. When doing the spectrum synthesis to estimate central line depths and select lines to include in our line lists, the abundances of some chemical elements were changed with respect to the Sun to mimic the abundance pattern typical of an $\mathrm{HgMn}$ star, e.g. $[\mathrm{Fe}]=+0.2,[\mathrm{Cr}]=+0.5$, $[\mathrm{Mg}]=-0.2,[\mathrm{Ti}]=+0.5$.

The effective temperature of each star, derived from the Strömgren photometric colors, was rounded to the nearest value in the model grid. A separate line mask was then created for each star. To exclude very weak lines from the calculation of LSD profiles, we required that the central depth of each line be $\geq 10 \%$ of the local continuum for the line to be included in the list. For the coolest star in our sample $\left(T_{\text {eff }} \approx 10500 \mathrm{~K}\right)$, this gave us 753 lines for the calculation of the LSD profiles, while for the hottest $\operatorname{star}\left(T_{\mathrm{eff}} \approx 14000 \mathrm{~K}\right)$ we used 344 lines. The resulting gain factor ranges from 5 to 17 , depending on the $S / N$ of observations and the $T_{\text {eff }}$ of the star.

For Stokes $I$, the line weights employed in the LSD analysis are assumed to be the central line depths provided by VALD. For the Stokes $V$ spectrum, we computed line weights using

$w=d z \frac{\lambda}{\lambda_{0}}$

where $d$ is the central line depth, $z$ is the effective Landé factor, $\lambda$ is the laboratory wavelength of the respective line, and $\lambda_{0}$ is a normalization wavelength, which we assumed to be equal to $4800 \AA$. In a few cases when Landé factors of the upper and/or lower atomic levels were missing in VALD, we used the LS-coupling scheme to compute them. In all other cases, Landé factors come from the quantum-mechanical calculations of Kurucz ${ }^{2}$. This way of assigning weights is considered standard (Donati et al. 1997).

We derived the Stokes $I, V$, and null LSD profiles using a code written by one of us (Kochukhov et al. 2010). All profiles were reconstructed using a velocity grid with a binsize of $0.8 \mathrm{~km} \mathrm{~s}^{-1}$, corresponding to the average pixel scale of HARPSpol spectra. The formal uncertainties in the LSD profiles were obtained by propagating the variance provided by the spectral reduction code for individual pixels. To account for possible under- or over-estimation of the error bars and for the eventual failure of the LSD assumptions, we scaled the uncertainties in the calculated LSD profiles to achieve reduced chi-square values of 1.0, in the same manner as described by Wade et al. (2000). In Fig. 2, we show an example of the LSD spectra obtained for the sharp-lined HgMn star HD 71066. This is the object for which we achieved our smallest uncertainty for the magnetic field diagnostics.

\subsection{Magnetic field measurements}

The mean longitudinal magnetic field, $\left\langle B_{z}\right\rangle$, was inferred from LSD profiles. Prior to the magnetic field measurements, we renormalize each LSD profile by a constant factor to obtain the correct continuum normalization. The displacement of the continuum is caused by the contribution of numerous unaccounted for weak blends in the LSD I profile, resulting in a slightly lower continuum level. The scaling of LSD $I, V$, and null profiles was done consistently for each star. Renormalized LSD profiles were then used to estimate the longitudinal magnetic using

$\left\langle B_{z}\right\rangle=-7.14 \times 10^{6} \frac{\int V\left(v-v_{0}\right) \mathrm{d} v}{\int(1-I) \mathrm{d} v}$,

where $V$ corresponds to the LSD circular polarization spectrum and $I$ corresponds to the LSD intensity profile, $v$ is the velocity, and $v_{0}$ is the velocity of the center-of-gravity of the LSD Stokes I profile. The constant factor in the right hand side of Eq. (2) includes $\lambda_{0}$ the normalization wavelength of the LSD profile, equal to $4800 \AA$, and $z_{0}$ the Landé factor of the LSD profile, equal to 1 according to our definition in Eq. (1). The resulting

${ }^{2}$ http://kurucz.harvard.edu/linelists.html 


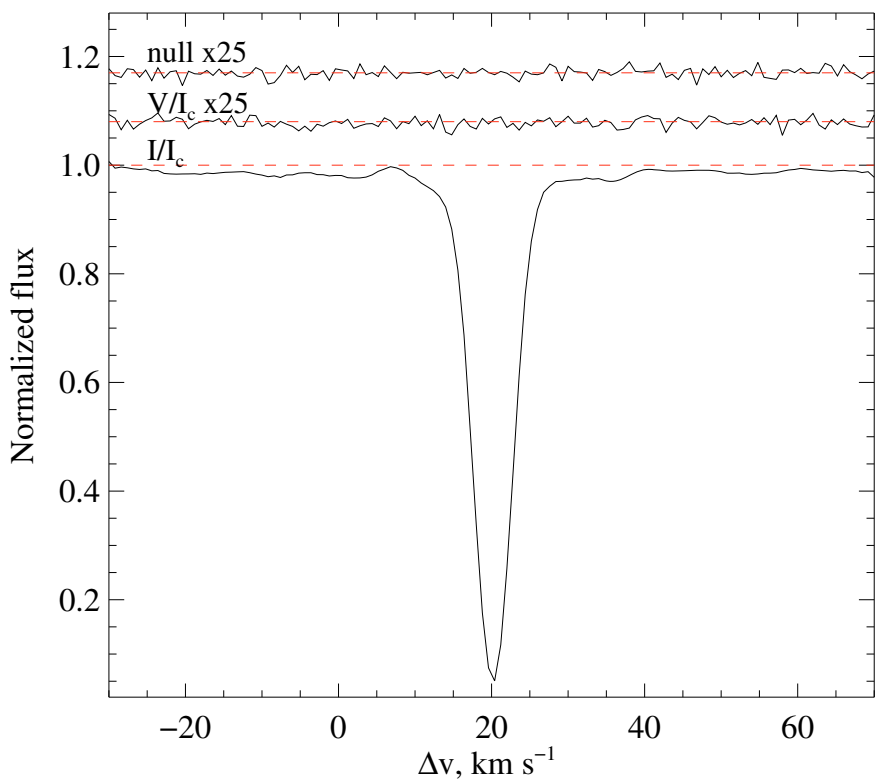

Fig. 2. The resulting LSD profiles for HD 71066. For display purposes, LSD $V$ and null profiles are shifted upwards by 1.08 and 1.17 , respectively, and multiplied by 25 .

values of $\left\langle B_{z}\right\rangle$ are in Gauss. The uncertainties in the $\left\langle B_{z}\right\rangle$ measurements are calculated by propagation of the LSD profile uncertainties.

The integration limits in Eq. (2) were chosen on the basis of the LSD I profile for each star. We note that the choice of integration limits can be quite important for the resulting $\left\langle B_{z}\right\rangle$ and its errors bar, especially for stars with $v_{\mathrm{e}} \sin i \lesssim 25 \mathrm{~km} \mathrm{~s}^{-1}$. The integration limits must be selected symmetrically with respect to the core of the LSD I profile. They should not exclude the wings of the profile but, at the same time, should not extend too far into the continuum, which contains no useful polarization signal. We find that for very slowly rotating stars the sensitivity of our $\left\langle B_{z}\right\rangle$ measurements can be degraded by $5-7 \mathrm{G}$ if the integration limits are changed by $\sim 3 \mathrm{~km} \mathrm{~s}^{-1}$. In this case, we carefully chose the integration limits to reach the highest precision of the longitudinal field determination. In the case of more rapid rotators, the definition of the measurement window is less important, so the integration limits can be set with the precision of $\sim 5 \mathrm{~km} \mathrm{~s}^{-1}$.

The same measurement procedure was also applied to the LSD profiles calculated from the null spectrum to test whether a real polarization signature is contained in the stellar spectrum or produced by spurious polarization. In general, the uncertainties in the longitudinal magnetic fields determined from the Stokes $V$ and null spectra are in excellent agreement and in none of the cases do we derive significant longitudinal field from the null profile. This result confirms the quality of our observations and analysis and suggests the absence of noticeable spurious polarization.

We measured magnetic fields for 41 objects. Since the target list includes SB2 systems with the contribution of both stars clearly being visible in the LSD profiles, we also measured magnetic fields in six secondary components. Most of them are Am stars (Ryabchikova 1998), except for HD 27376, whose secondary has a $\mathrm{Hg}$ peculiarity. There are $68\left\langle B_{z}\right\rangle$ measurements in total, as some of the stars were observed more than once.

For all stars, our measured $\left\langle B_{z}\right\rangle$ values are below the $3 \sigma$ level. Similarly, we do not detect $\left\langle B_{z}\right\rangle$ using the null spectra. The best precision we achieved for our longitudinal magnetic
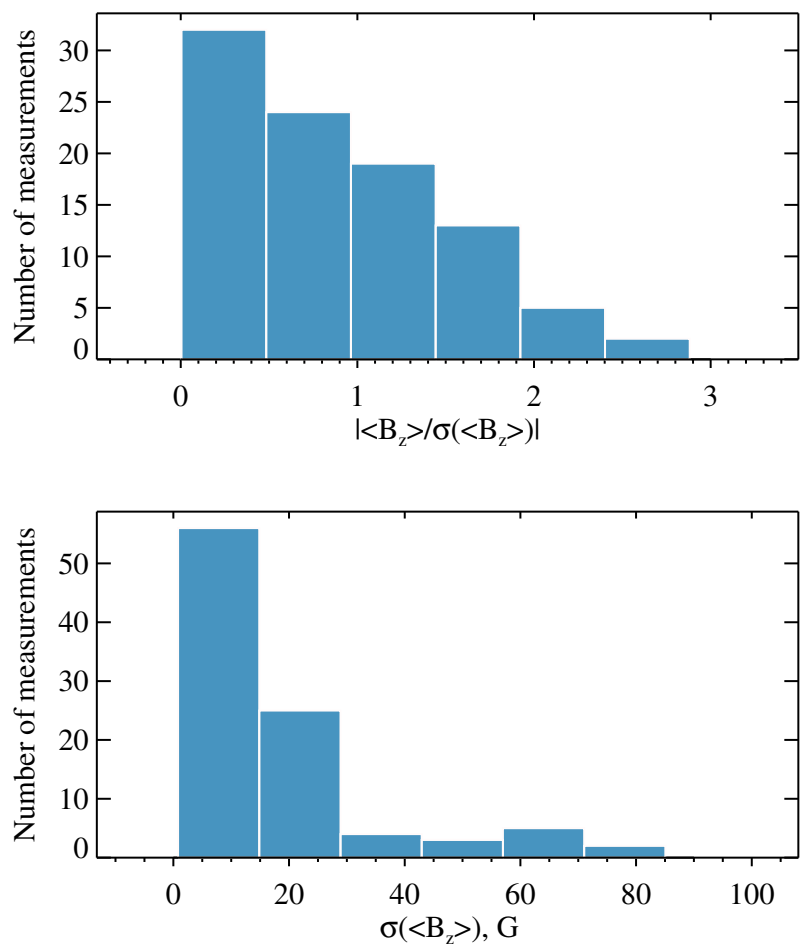

Fig. 3. The upper panel: the distribution of the measured longitudinal field divided by its error. The lower panel: the distribution of longitudinal magnetic field error.

field measurements was for HD 71066, for which our uncertainty is just $0.81 \mathrm{G}$. Figure 3 presents a statistical summary of our results, excluding measurements of non- $\mathrm{HgMn}$ stars. The upper panel shows the distribution of the ratio of $\left\langle B_{z}\right\rangle$ to its uncertainty. The lower panel illustrates the overall distribution of the longitudinal field uncertainties. The sensitivity of the majority of our measurements lies in the range of 1-20 G, which is much better than in previous studies of $\mathrm{HgMn}$ stars.

For certain magnetic field configurations, the first moment of the LSD Stokes $V$ profile appearing in Eq. (2) may be zero, while the profile itself manifests a magnetic signature. To examine these situations, we employed the false alarm probability (FAP) analysis (Donati et al. 1997). It is a $\chi^{2}$ probability statistic that estimates the probability of a given signal being produced by random noise. We employed FAP analysis as a tool for detecting a magnetic signal in the LSD Stokes $V$ spectra, assuming no detection if FAP $>10^{-3}$, marginal detection if $10^{-3} \leq \mathrm{FAP} \leq 10^{-5}$, and definite detection if FAP $<10^{-5}$. We report no detection of any signatures indicative of a magnetic field in any of the $41 \mathrm{HgMn}$ stars we observed. There is also no evidence of the coherent signal in any of the null spectra. For one star, HD 178065, the FAP analysis of the LSD Stokes $V$ yielded a marginal detection. This result might be a statistical fluctuation since the measured FAP is close to the (arbitrary) boundary between no detection and marginal detection. No longitudinal magnetic field was detected for this $\operatorname{star}\left(\left\langle B_{z}\right\rangle=-2.26 \pm 2.08 \mathrm{G}\right)$.

A complete summary of our analysis of the circular polarization spectra of HgMn stars is presented in Table 2. In the columns from left to right, we list the stellar HD number, designation of a binary component (A - for primary, B - for secondary and $\mathrm{AB}$ - for one measurement of the blended components of HD 27376). This is followed by columns containing the Julian date of observation, the $S / N$ ratio of the observed Stokes $V$ spectrum and of the reconstructed LSD Stokes $V$ profile, the mean 
longitudinal magnetic field derived from the Stokes $V$ and null spectra, together with the respective uncertainties. In a few cases, polarization measurements were based on two sub-exposures only, which did not allow us to compute the null spectrum. The uncertainties correspond to $1 \sigma$ confidence levels. The last column gives the FAP for the presence of a signal in the LSD Stokes $V$ profile. All LSD Stokes $I, V$, and null spectra are presented in Fig. 5.

\section{Discussion}

As already mentioned, previous studies of magnetic fields in $\mathrm{HgMn}$ stars did not detect longitudinal fields stronger than 29-100 G, and in one particular case the limits were 6-19 G. These $1 \sigma$ error bars are not low enough to exclude the presence of the longitudinal fields weaker than $\sim 100 \mathrm{G}$, which could result from dynamically important local magnetic field structures covering only a fraction of the stellar surface. Thus, our understanding of the magnetic characteristics of these stars was fundamentally incomplete. To achieve a more detailed picture of the magnetic properties of $\mathrm{HgMn}$ stars, we have studied a large sample of such stars with the most stable and modern spectropolarimeter available.

Our analysis of the Stokes $V$ spectra showed no evidence of any polarimetric signal, setting $3 \sigma$ upper limits on the longitudinal field present of 3-30 G for the majority of the stars we observed. For one sharp-lined $\mathrm{HgMn}$ star, we obtained an uncertainty of $\sigma_{B_{z}}<1 \mathrm{G}$, which is better than previously achieved with a single observation of any early-type star. In addition to obtaining null results for longitudinal magnetic field measurements, we analyzed the Stokes $V$ LSD profiles themselves, finding no evidence of complex polarization signatures. Thus, we conclude that mercury-manganese stars do not possess strong, but topologically complex, magnetic fields. If we restrict ourselves to a dipolar magnetic field topologies, the dipole strength can be roughly estimated as $B_{\mathrm{d}} \sim 3\left\langle B_{z}\right\rangle$. On the basis of our longitudinal field measurements, we conclude that the HgMn stars do not possess dipolar fields stronger than $3-30 \mathrm{G}$.

It is important to evaluate our results in the context of the possible role of magnetic fields in the recently discovered spot formation on $\mathrm{HgMn}$ stars. Although there are no detailed models describing the process of the interaction between magnetic fields and the plasma, it is understood (e.g., Wade et al. 2006; Aurière et al. 2007) that this interaction becomes important when the strength of magnetic field exceeds the equipartition limit, defined by Wade et al. (2006) as $B_{\text {eq }}=\sqrt{12 \pi P_{\text {gas }}}$. At this field strength, the energy of the magnetic field equals that of the gas.

We estimated the value of the equipartition magnetic field strength for atmosphere models with effective temperatures of $T_{\text {eff }}=10000 \mathrm{~K}$ and $T_{\text {eff }}=15000 \mathrm{~K}$, representing the range covered by our sample of $\mathrm{HgMn}$ stars. Using thermodynamic parameters tabulated in these models and assuming the ideal gas law, we calculated the equipartition field as a function of the optical depth in the continuum. The result is presented in Fig. 4. From this figure, we can see that the value of $B_{\text {eq }}$ at $\log \tau_{5000}=-0.5$ is $\sim 200 \mathrm{G}$ for the stars of both low and high effective temperatures. The value of $B_{\text {eq }}$ then decreases to $100 \mathrm{G}$ at $\log \tau_{5000}=-2$ for $T_{\text {eff }}=10000 \mathrm{~K}$ and to $60 \mathrm{G}$ for $T_{\text {eff }}=$ $15000 \mathrm{~K}$. The majority of spectral lines used in our measurements form over a range in optical depth from $\log \tau_{5000}=-0.5$ to -2 . In this interval, the values of $B_{\text {eq }}$ are much higher than our upper limits for the organized magnetic field on the stellar surface. Thus, it appears that there are no magnetic fields on the surfaces of HgMn stars that could lead to the formation of

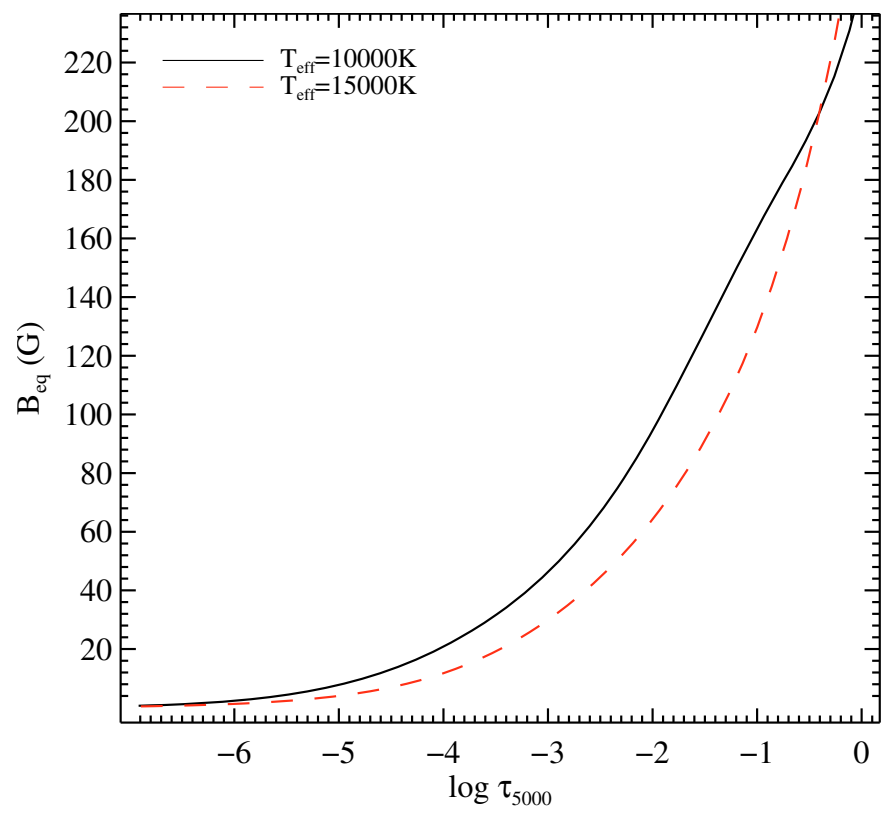

Fig. 4. The equipartition magnetic field as a function of the optical depth. The solid line corresponds to the cooler model and the dashed one to the hotter.

chemical spots. These conclusions apply to the fields stronger than $\sim 10 \mathrm{G}$ probed by our study. On the other hand, we cannot exclude the presence of complex magnetic fields with a field strength of $\sim 1 \mathrm{G}$ in surface spots, similar to the tangled magnetic topology suggested for Vega (Petit et al. 2010).

These results lead to the conclusion that there exists a real dichotomy between $\mathrm{HgMn}$ and magnetic Ap stars. The latter show chemical inhomogeneities in the presence of dipolar-like magnetic fields stronger than $300 \mathrm{G}$. This limit is determined by the field strength that can withstand shearing by differential rotation and survive at the surfaces of early-type stars (Aurière et al. 2007). In contrast to magnetic Ap stars and despite a similarity between their fundamental parameters, $\mathrm{HgMn}$ stars clearly do not have magnetic fields reaching this limit. The surface chemical structures in mercury-manganese stars must therefore form under a mechanism fundamentally different from magneticallydriven spot formation in hot Ap or cool active stars.

A detailed Doppler imaging study of the surface structures on the brightest HgMn star, $\alpha$ And, carried out by Kochukhov et al. (2007) using observations collected over a period of seven years led to the remarkable discovery of $\mathrm{Hg}$ spot evolution. This is very different from the spotted early-type magnetic stars, in which the morphology of spots remains steady over tens of years. That no magnetic field was detected in $\alpha$ And (Wade et al. 2006) makes the explanation of the spot formation and evolution a very challenging task. Kochukhov et al. (2007) suggested that mercury atoms are concentrated in a thin layer where the radiation pressure and gravitational force compensate each other. The observed changes in the spot morphology are then ascribed to hydrodynamical instabilities, upsetting the fine force balance in the $\mathrm{Hg}$ cloud. The physics of this intricate process and its relation to the binarity, stellar rotation, and dynamic atomic diffusion effects is yet to be explored.

Despite having been frequent targets of detailed abundance analyses over more than half a century, HgMn stars were recognized as spotted variables only recently. To date, seven spotted $\mathrm{HgMn}$ stars have been found. It is uncertain how many more of these stars will be discovered. Prior to the formulation of exotic 
theories of non-magnetic chemical spot formation, it is important to determine a complete picture of the occurrence rate of chemical inhomogeneities on the surface of $\mathrm{HgMn}$ stars. If spots are found in only a small fraction of these objects, it may be that the observed structure formation is associated with a certain stage of stellar evolution or a certain configuration of the binary system. On the other hand, discovery of spots in the majority of HgMn stars would indicate the presence of a universal hydrodynamical process, not anticipated in current stellar models.

We suggest that in-depth studies of selected spotted $\mathrm{HgMn}$ stars as well as a general searches for spectral line variability in all accessible objects of this type will provide sufficient observational constraints to develop a theory of non-magnetic spot formation. Analysis of known spotted stars can yield information on the timescales of spot evolution and, possibly, on the heights of these inhomogeneities relative to the typical line formation regions. It is also important to continue searching for weak magnetic fields in spotted $\mathrm{HgMn}$ stars as previously done by Wade et al. (2006) for $\alpha$ And and by Folsom et al. (2010) for AR Aur. On the other hand, a line profile variability survey of a large sample of $\mathrm{HgMn}$ stars will help us to uncover dependences on stellar parameters, rotation rate, or binarity, possibly identifying a correlation of one of these parameters with the presence of spots. In subsequent studies of $\mathrm{HgMn}$ stars, we plan to carry out both detailed studies of individual objects and perform variability surveys to find new spectroscopically variable stars.

Acknowledgements. Authors thank the referee Pascal Petit for his valuable comments. V.M. and N.P. thank the European Southern Observatory (ESO) engineering team for excellent support during the commissioning of HARPSpol. O.K. is a Royal Swedish Academy of Sciences Research Fellow, supported by grants from Knut and Alice Wallenberg Foundation and Swedish Research Council.

\section{References}

Abt, H. A., \& Morrell, N. I. 1995, ApJS, 99, 135

Adelman, S. J., Malanushenko, V., Ryabchikova, T. A., \& Savanov, I. 2001, A\&A, 375, 982
Adelman, S. J., Gulliver, A. F., Kochukhov, O. P., \& Ryabchikova, T. A. 2002, ApJ, 575, 449

Aurière, M., Wade, G. A., Silvester, J., et al. 2007, A\&A, 475, 1053

Aurière, M., Wade, G. A., Konstantinova-Antova, R., et al. 2009, A\&A, 504, 231

Auriere, M., Wade, G. A., Lignieres, F., et al. 2010, A\&A, 523, A40

Bagnulo, S., Landolfi, M., Landstreet, J. D., et al. 2009, PASP, 121, 993

Briquet, M., Korhonen, H., González, J. F., Hubrig, S., \& Hackman, T. 2010, A\&A, 511, A71

Donati, J., Semel, M., Carter, B. D., Rees, D. E., \& Collier Cameron, A. 1997, MNRAS, 291, 658

Dworetsky, M. M. 1993, in Peculiar versus Normal Phenomena in A-type and Related Stars, ed. M. M. Dworetsky, F. Castelli, \& R. Faraggiana, IAU Colloq., 138, ASP Conf. Ser., 44, 1

Folsom, C. P., Kochukhov, O., Wade, G. A., Silvester, J., \& Bagnulo, S. 2010, MNRAS, 407, 2383

Hubrig, S., González, J. F., Savanov, I., et al. 2006, MNRAS, 371, 1953

Jeffers, S. V., \& Donati, J. 2008, MNRAS, 390, 635

Kochukhov, O. 2004, in The A-Star Puzzle, ed. J. Zverko, J. Ziznovsky, S. J. Adelman, \& W. W. Weiss, IAU Symp., 224, 433

Kochukhov, O., Piskunov, N., Sachkov, M., \& Kudryavtsev, D. 2005, A\&A, 439, 1093

Kochukhov, O., Adelman, S. J., Gulliver, A. F., \& Piskunov, N. 2007, Nat. Phys., 3, 526

Kochukhov, O., Makaganiuk, V., \& Piskunov, N. 2010, A\&A, 524, A5

Kupka, F., \& Bruntt, H. 2001, J. Astron. Data, 7, 8

Lignières, F., Petit, P., Böhm, T., \& Aurière, M. 2009, A\&A, 500, L41

Lüftinger, T., Fröhlich, H., Weiss, W. W., et al. 2010, A\&A, 509, A43

Mayor, M., Pepe, F., Queloz, D., et al. 2003, The Messenger, 114, 20

Moon, T. T., \& Dworetsky, M. M. 1985, MNRAS, 217, 305

Petit, P., Lignières, F., Wade, G. A., et al. 2010, A\&A, 523, A41

Piskunov, N. E., \& Valenti, J. A. 2002, A\&A, 385, 1095

Piskunov, N. E., Kupka, F., Ryabchikova, T. A., Weiss, W. W., \& Jeffery, C. S. 1995, A\&AS, 112, 525

Pourbaix, D., Tokovinin, A. A., Batten, A. H., et al. 2004, A\&A, 424, 727

Renson, P., \& Manfroid, J. 2009, A\&A, 498, 961

Ryabchikova, T. 1998, Contributions of the Astronomical Observatory Skalnate Pleso, 27, 319

Shorlin, S. L. S., Wade, G. A., Donati, J., et al. 2002, A\&A, 392, 637

Shulyak, D., Tsymbal, V., Ryabchikova, T., Stütz, C., \& Weiss, W. W. 2004, A\&A, 428, 993

Snik, F., Jeffers, S., Keller, C., et al. 2008, in SPIE Conf. Ser., 7014, 22

Wade, G. A., Donati, J., Landstreet, J. D., \& Shorlin, S. L. S. 2000, MNRAS, 313,823

Wade, G. A., Aurière, M., Bagnulo, S., et al. 2006, A\&A, 451, 293

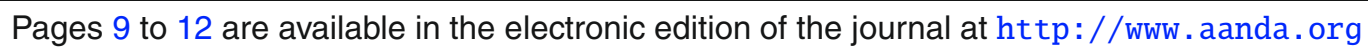


V. Makaganiuk et al.: The search for magnetic fields in mercury-manganese stars
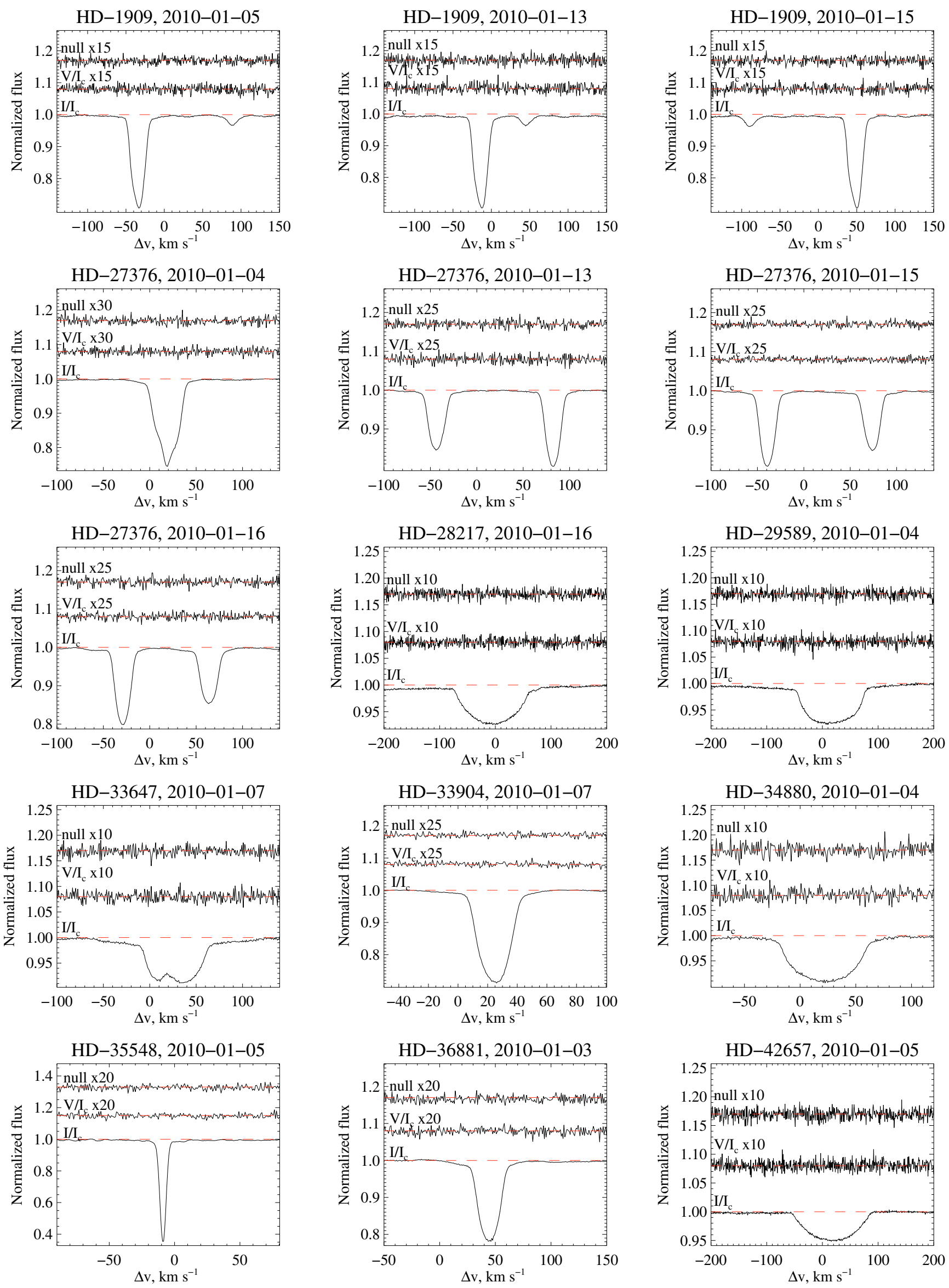

Fig. 5. From bottom to top, each panel shows the LSD profiles of Stokes $I, V$, and null spectrum (if available) for all $\mathrm{HgMn}$ stars in our survey. 
A\&A 525, A97 (2011)
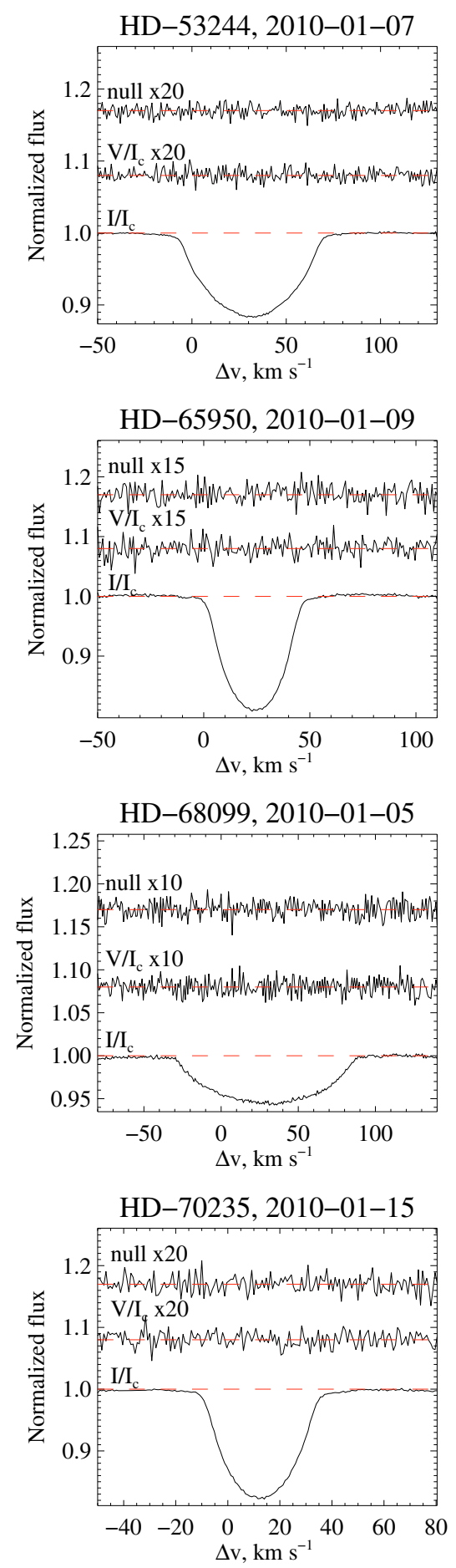

HD-71833, 2010-01-15

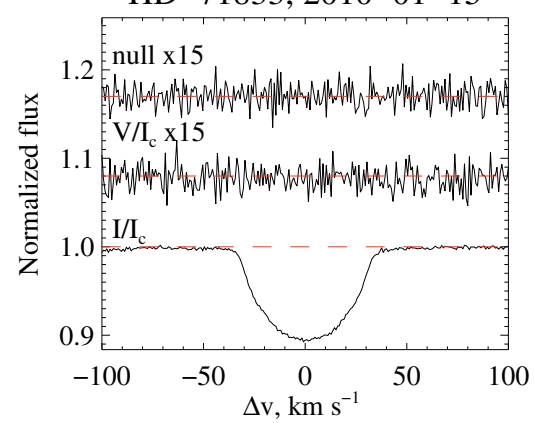

Fig. 5. continued.
HD-53929, 2010-01-03

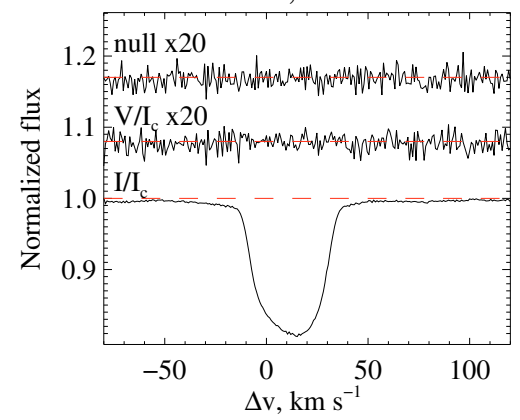

HD-65950, 2010-01-14

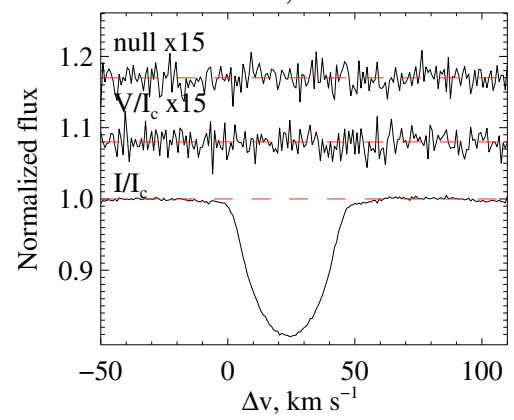

HD-68099, 2010-01-15

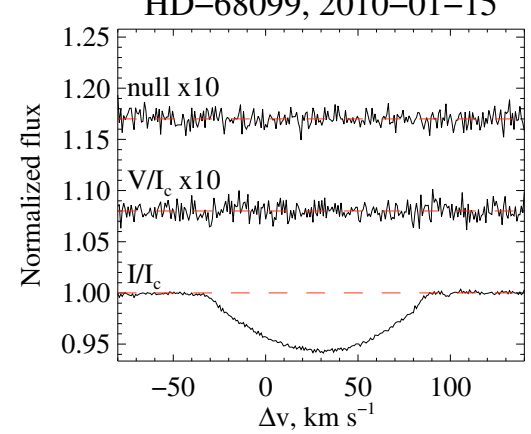

HD-71066, 2010-01-08

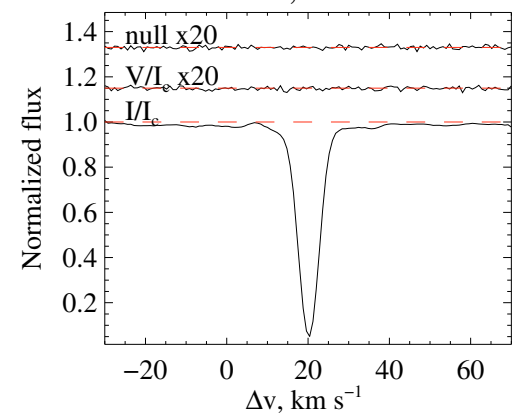

HD-72208, 2010-01-05

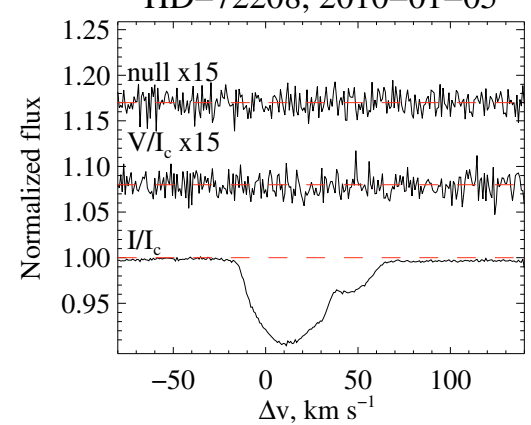

HD-63975, 2010-01-07

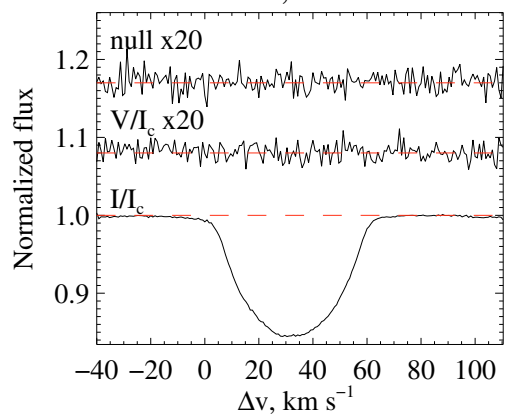

HD-65950, 2010-01-15

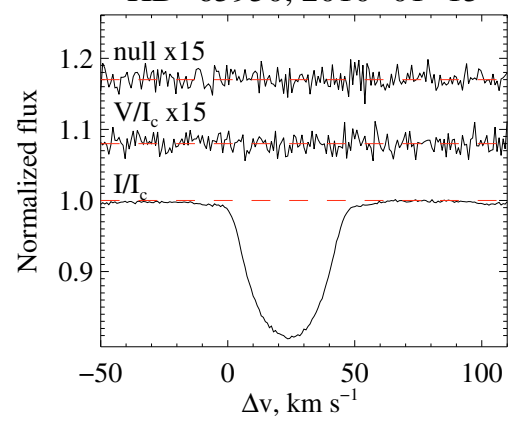

HD-70235, 2010-01-08

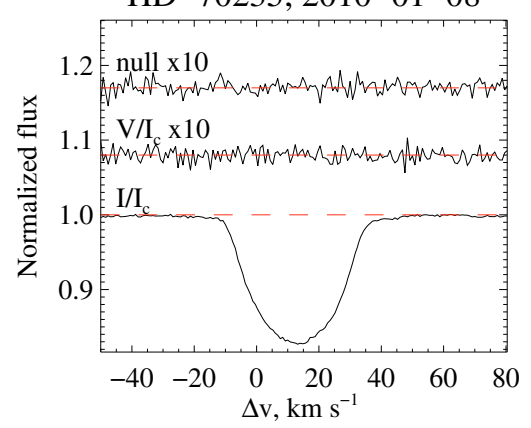

HD-71833, 2010-01-08

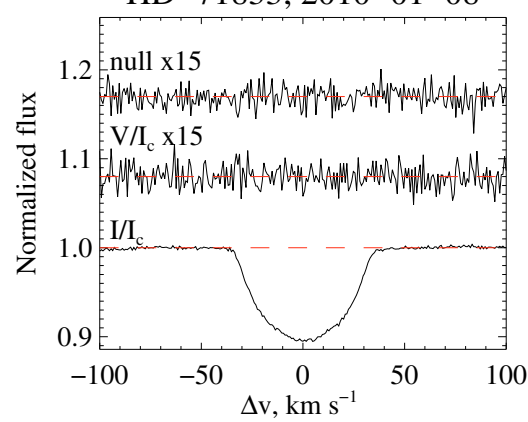

HD-72208, 2010-01-12

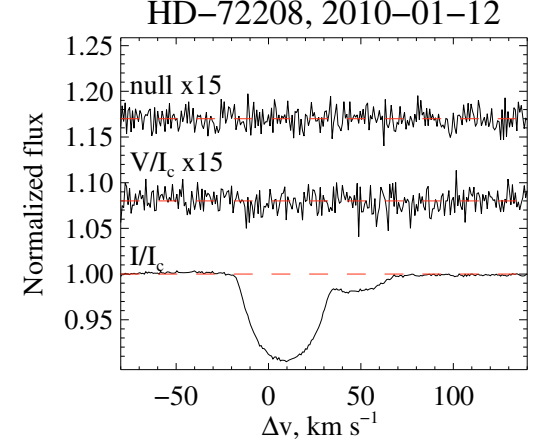


V. Makaganiuk et al.: The search for magnetic fields in mercury-manganese stars
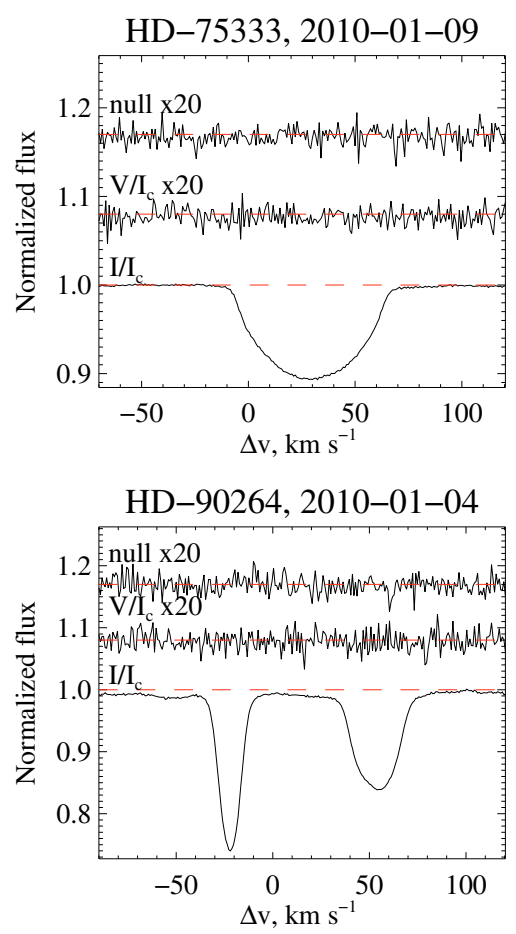

HD-106625, 2009-06-01
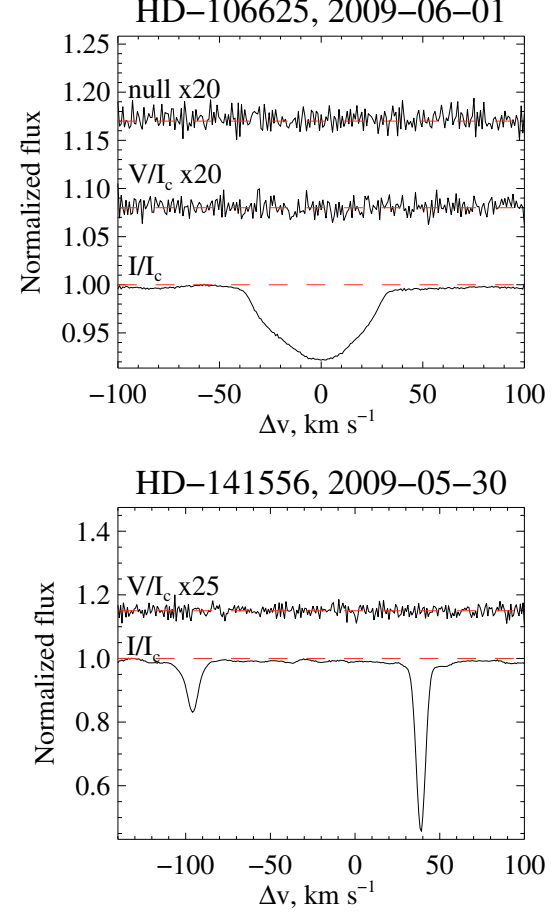

HD-158704, 2010-04-29

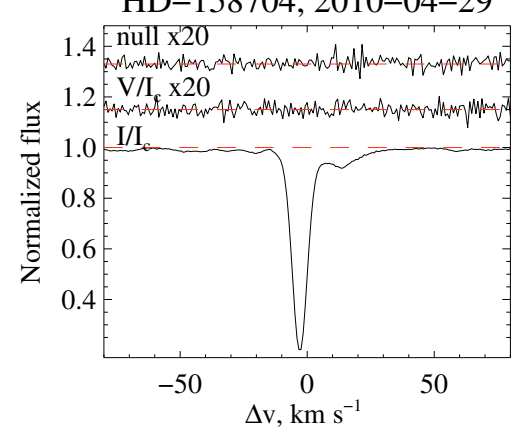

Fig. 5. continued.
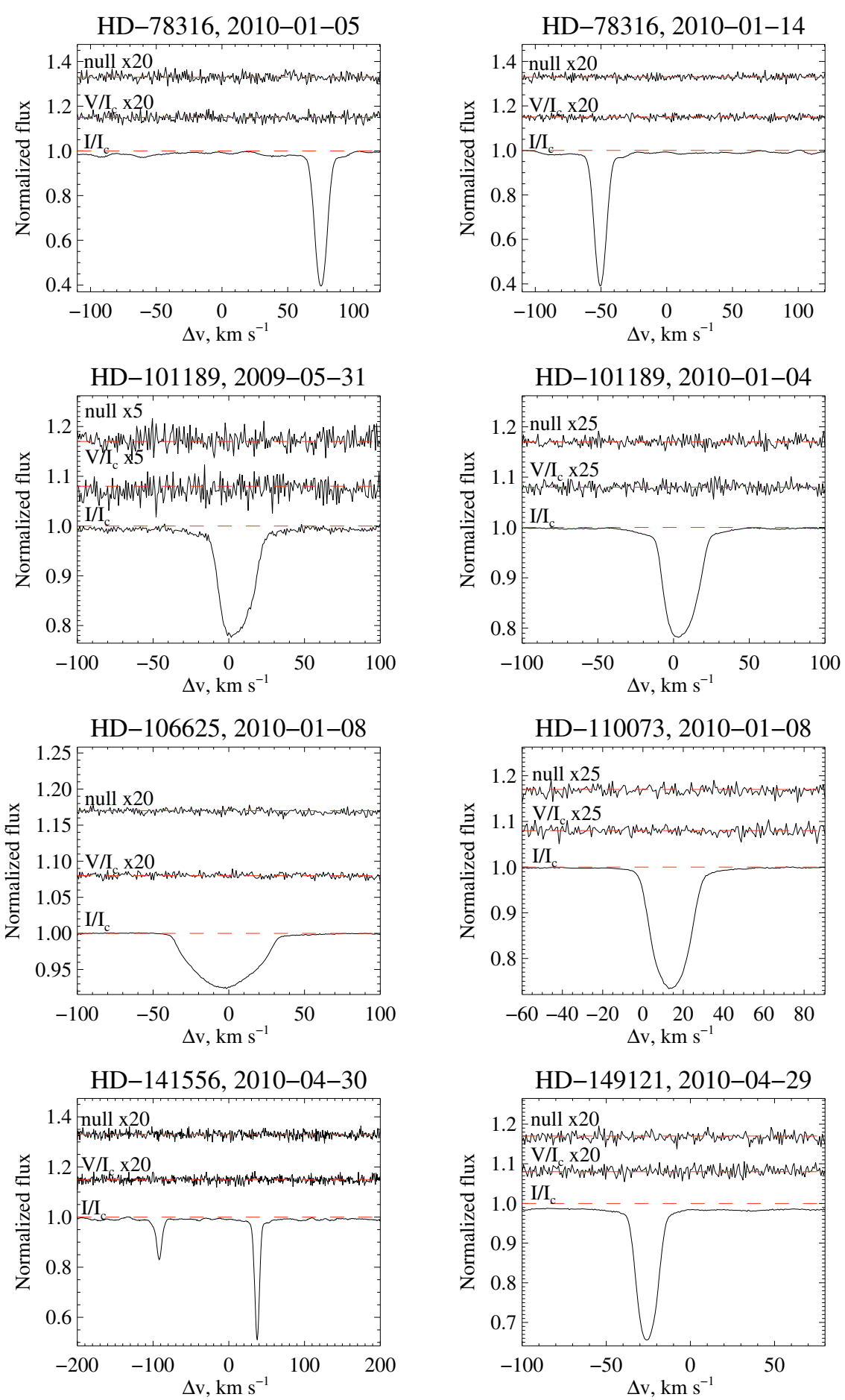

HD-165493, 2010-04-29

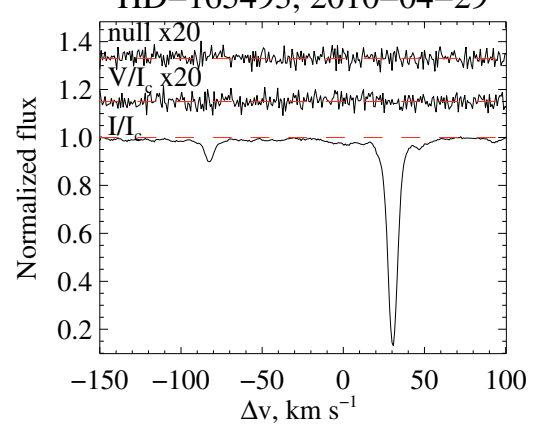

HD-175640, 2010-04-30

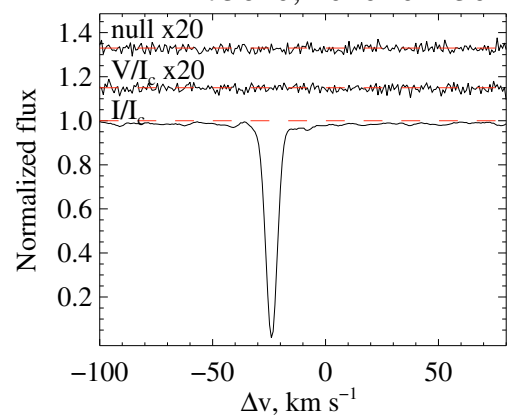


A\&A 525, A97 (2011)

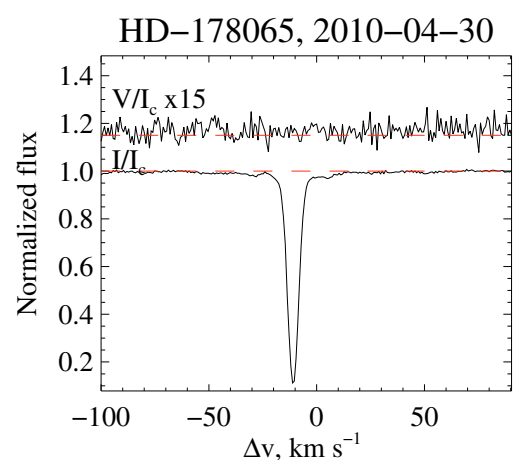

HD-186122, 2010-05-02

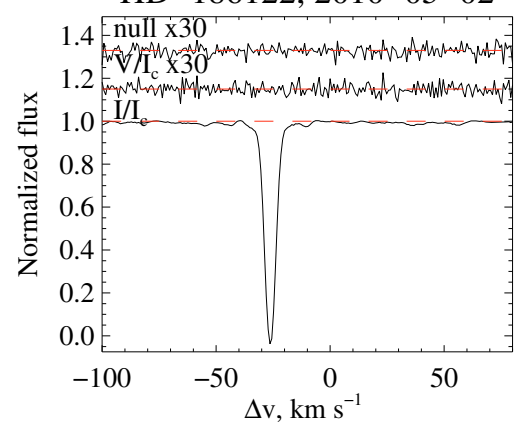

HD-193452, 2010-05-02

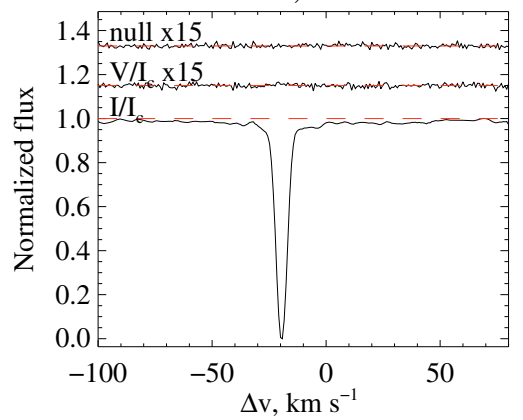

HD-202671, 2009-06-02

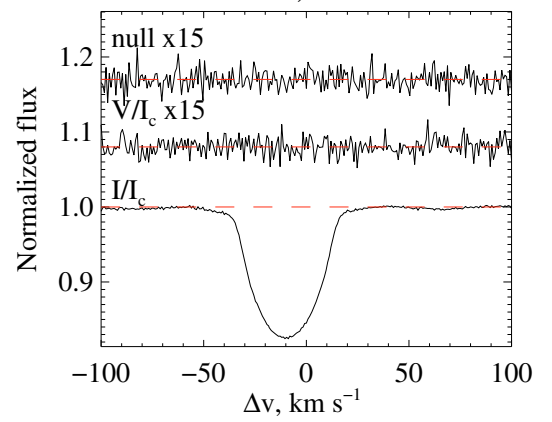

Fig. 5. continued.
HD-178065, 2010-05-02
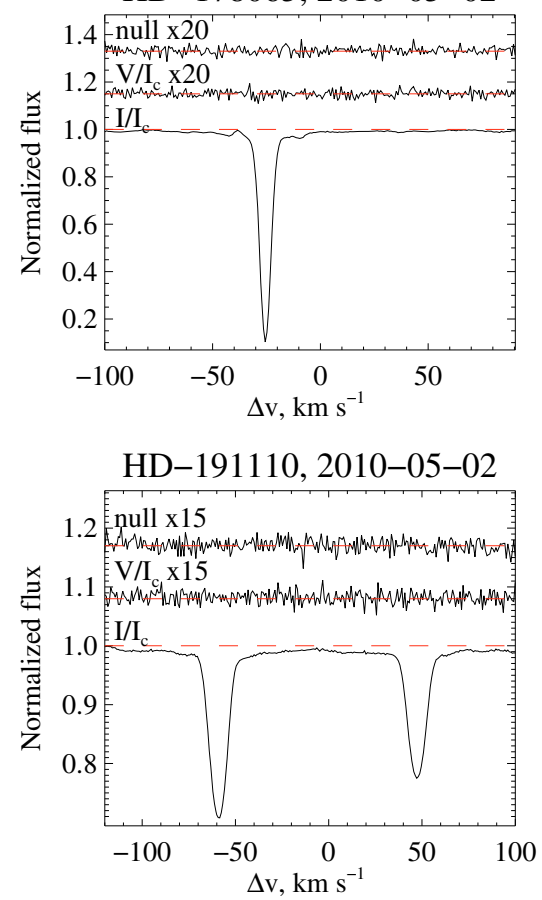

HD-194783, 2009-06-02

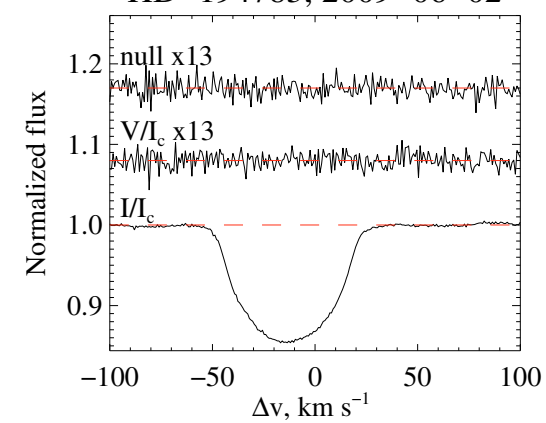

HD-211838, 2009-06-02

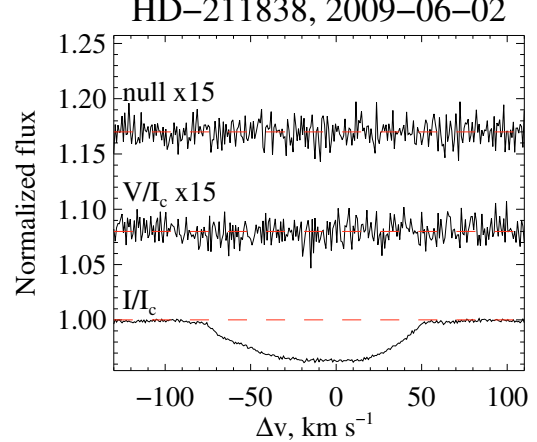

HD-179761, 2010-05-02
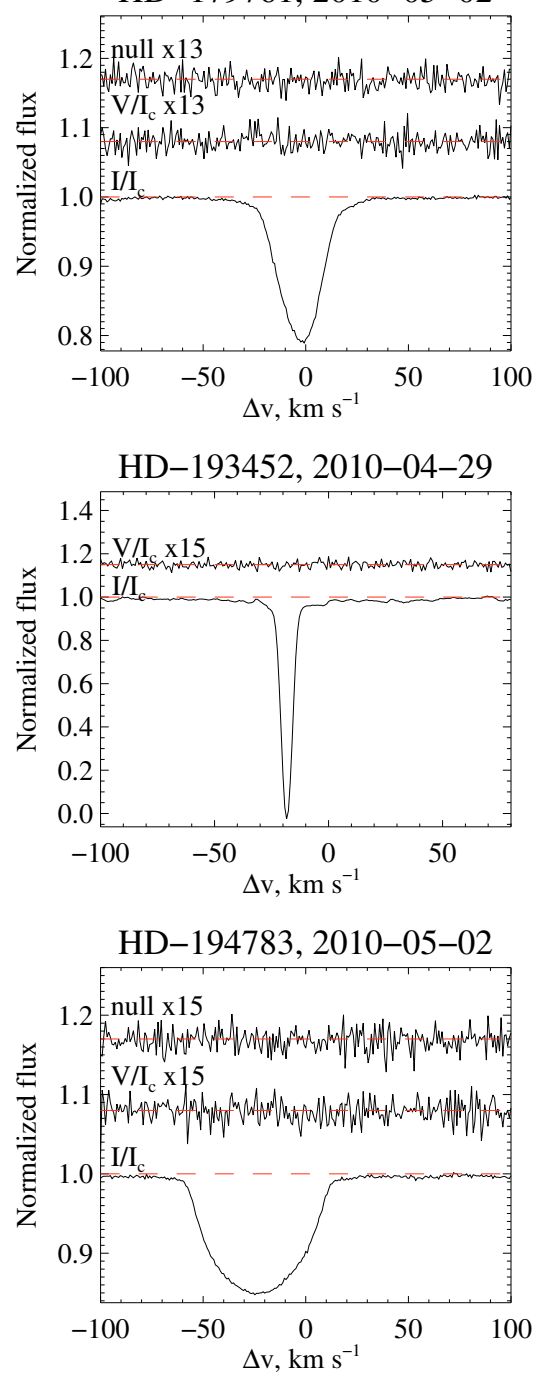

HD-221507, 2010-01-13

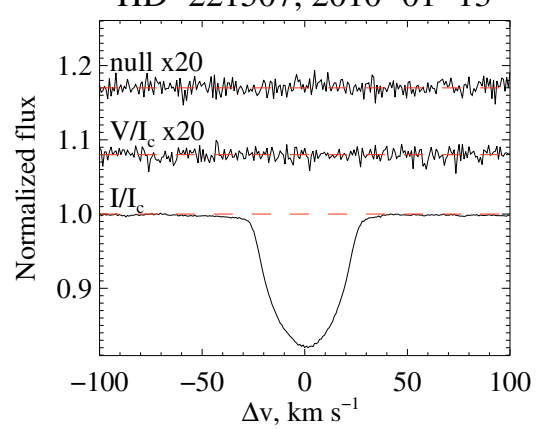

